\title{
Design of Center Cable Drill Rod for Coal Mine
}

\author{
Nanfei Yan \\ China Coal Technology and Engineering Group Xi'an Research Institute, Xi'an 710077, China \\ 502300500@qq.com
}

Keywords: Center cable. Signal transmission. Insulating device. Downhole motor Abstract .The center cable drill rod for coal mine can transmit electric power signals from hole top to bottom while establishing a two-way high-speed information channel between devices at hole top and bottom. This paper introduces the design of outer pipe and inner structure of the cable drill rod and researches critical factors influencing signal transmission. This key technology can ensure the stability and reliability of final signal transmission only when given full attention.

\section{Introduction}

Coal is a conventional source of energy, which is most widely distributed and cheapest and whose reserve is the greatest in the world ${ }^{[1]}$, and it is also a basic energy source in China. In recent years, driven by rapid growth of the national economy, rapid development of power, metallurgy, building material, chemical and other industries leads to significantly increased demand for coal and more consumption of coal each year. Coal production and consumption keep growing fast in China, but for a long time, gas disaster has been impacting the safety in coal mine production. Especially in highly gassy mines, besides high cost of gas control, failure of timely connection of gas control with raw coal production also restricts coal mine production ${ }^{[2]}$. Therefore, highly gassy mines strive to ease the contradiction between production and gas prevention \& control by highly efficient gas drainage technologies and advanced equipment so as to improve the economic benefit of the mine.

Draining coal seam gas through the borehole is a common and effective method used in China $^{[3]}$, and features rapid construction and flexible layout of drill site. However, low borehole depth and short distance of gas drainage result in short continuous working hours of coal cutter and low efficiency of coal cutting. In order to increase the borehole depth and improve the efficiency of coal cutting, there must be a complete set of equipment suitable for gas drainage through long borehole in Chinese coal mines and thereby ideal drainage can be achieved. Through years of study, CCTEG Xi'an Research Institute has developed a set of equipment and techniques for directional drilling along coal seam, which can make borehole depth reach above 1,000 m. This set of equipment has currently been put into coal mine production with high output and efficiency and has played an important role in the gas control for highly gassy mines ${ }^{[4]}$.

A key technology of the equipment for directional drilling along coal seam is the development of center cable drill rod. This type of drill rod ensures the signal transmission between the monitoring device at hole top and downhole equipment and reflects the dip angle and orientation angle of the borehole in real time, which makes the borehole track clearly visible and thereby ensures the operability of the technology of directional drilling along coal seam.

\section{Design of Outer Pipe of Center Cable Drill Rod}

Outer pipe is an important component of center cable drill rod. It should ensure not only the 
rigidity and enough stretching resistance upon strong pulling when borehole depth reaches 1,000 $\mathrm{m}$, but also the rod's capability of bearing sufficient toque in case of strong rotation of the rod at hole collapse. Moreover, the inner through hole of rod shall be big enough, for the inner through hole should not only set the signal transmission device but also ensure the throughput of high-pressure flushing fluid. If the inner through hole is not big enough to ensure the flow rate of high-pressure medium, the helicoid hydraulic motor will not be driven, the downhole motor will not work and thus the drilling process will not be completed. Therefore, big enough inner through hole for the rod is a necessary condition for ensuring the directional drilling process.

\section{Structure design of outer pipe}

In order to ensure the inner through hole of the rod, we designed an initial scheme according to which seamless steel tube is directly threaded, as shown in Fig. 1. As the wall of the rod is relatively thin, the wall at the screw thread of the rod is even thinner after threading. Though big through hole is ensured in this way, calculation shows that the strength of this rod is extremely low and cannot meet the strength requirement for $1,000 \mathrm{~m}$ deep borehole at all. Therefore, we designed a friction-welded drill rod, whose structure is shown in Fig. 2.

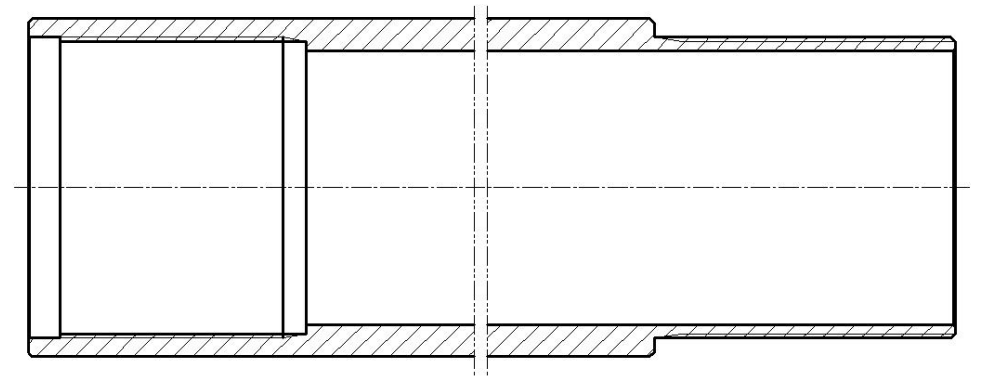

Fig. 1 Structure Diagram of Steel Tube Drill Rod

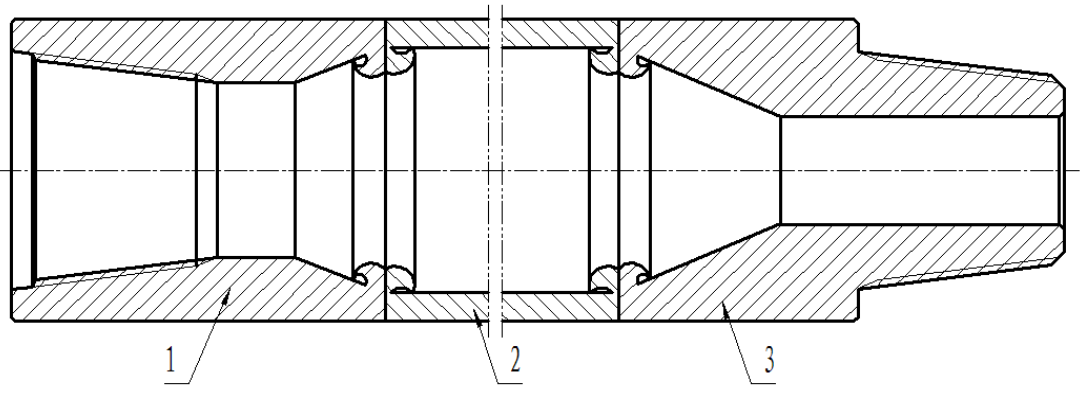

Fig. 2 Structure Diagram of Friction-welded Drill Rod

1 and 3 refer to joints and 2 to seamless pipe body in Fig. 2. Joints and seamless pipe body are connected by friction welding, and this kind of rod ensures the overall strength of the rod by properly increasing the wall thickness of thread at the joints. Besides, the thread must be abrasion resistant to a certain extent because the rod is subject to frequent screwing and unscrewing. As the rod is just threaded at the joints, material for the joints must be that is highly resistant to abrasion.

\section{Thermal treatment of outer pipe}

In order to ensure the strength of the rod, we gave it several times of thermal treatment. The first step is hardening and tempering of the joints to make their strength reach $H R C \geq 30$. After hardening and tempering, the screw thread is not only resistant to abrasion but also strong and tough. The second step is thermal treatment of friction welding seam, which is one-time tempering treatment. When the rod is welded, high-speed friction and plastic deformation in the welding seam area generated a high temperature of about $1200^{\circ} \mathrm{C}-1300^{\circ} \mathrm{C}$, accompanied by the transformation of austenite structure. After upsetting, heat conduction of the cool metal on both sides of the welding 
seam made the welding seam area which had undergone macrostructure phase transformation rapidly cooled and quenched. This area is not only hard (up to HB520), with oversized crystal grain and microscopic structure, but also bears internal stresses to different degrees, with embrittlement phenomena of welding such as superheated brittlement and martensite transformation brittlement, making the structure and performance of the weld zone in as-welded condition hard to meet the requirements. Therefore, the weld zone of the rod after welding must undergo tempering thermal treatment to make the structure of the crystalline phase of the welding seam reasonable. In this way, the strength of the welding seam is ensured at no lower than $90 \%$ of that of the base metal, and the overall strength of the rod is also ensured. At last, since thread gluing is easy to occur to this rod due to large diameter of the inner through hole and small thread taper, we needed to give high-frequency quenching thermal treatment to the screw thread, which significantly enhanced the strength of the screw thread and ensured not only the abrasive resistance of the screw thread but also its resistance to thread gluing.

\section{Pilot production of outer pipe}

Based on the structure of the rod, we designed processing techniques with consideration given to the drilling technology and the high strength and roughness of large-diameter long-borehole rod. We determine the two key procedures of friction welding and thermal treatment of the rod, which have influence on rod strength and roughness, as special procedures, and joint processing before friction welding and final thread machining as two critical procedures. The quality of rod will be fully ensured if these key links are well completed in the manufacture of the rod. Secondly, we adopted proper equipment for rod machining according to the technological line, including such major equipment as full-automatic band sawing machine, center lathe, CNC lathe, friction welding machine, continuous quenching furnace and box-type tempering furnace. The quality of rod is ensured with such equipment. Especially, machining of rod thread must be completed with the CNC lathe, for the thread not only should be highly precise but also be interchangeable to a certain extent. Center lathe is not able to ensure such precision and interchangeability and its efficient is also low. Moreover, in order to ensure the precision of thread form, we adopted imported molding cutter, which is not only abrasion-resistant but also machines thread with high degree of finish and high efficiency. In this way, the precision of thread and the interchangeability of rod are ensured.

\section{Design of Inner Structure of Center Cable Drill Rod}

As center cable drill rod should not only ensure signal transmission but also passing of high-pressure flushing medium inside the rod, higher requirements are imposed on its inner structure. First, signal transmission device should be set at the inner center along the axial line. Second, a layer of insulating device should be set outside the signal transmission device so as to ensure stable signal transmission and prevent aqueous medium from entering into the transmission device and impacting signal transmission. In view of this, we designed the structure of rod, as shown in Fig. 3. The signal transmission device consists of conductor pin joint 3, copper conductor 10, conductor box joint 8 and variable-diameter spring 9. The insulating device consists of insulating pin joint 2, conduit 5 and insulating box joint 7 . The insulating device is fixed at the axial line of outer pipe 1 by the locating retainer rings 4 at both ends and stabilizer 6 in the middle. The high-pressure aqueous medium flows through the ringlike gap between the insulating device and the outer pipe. 

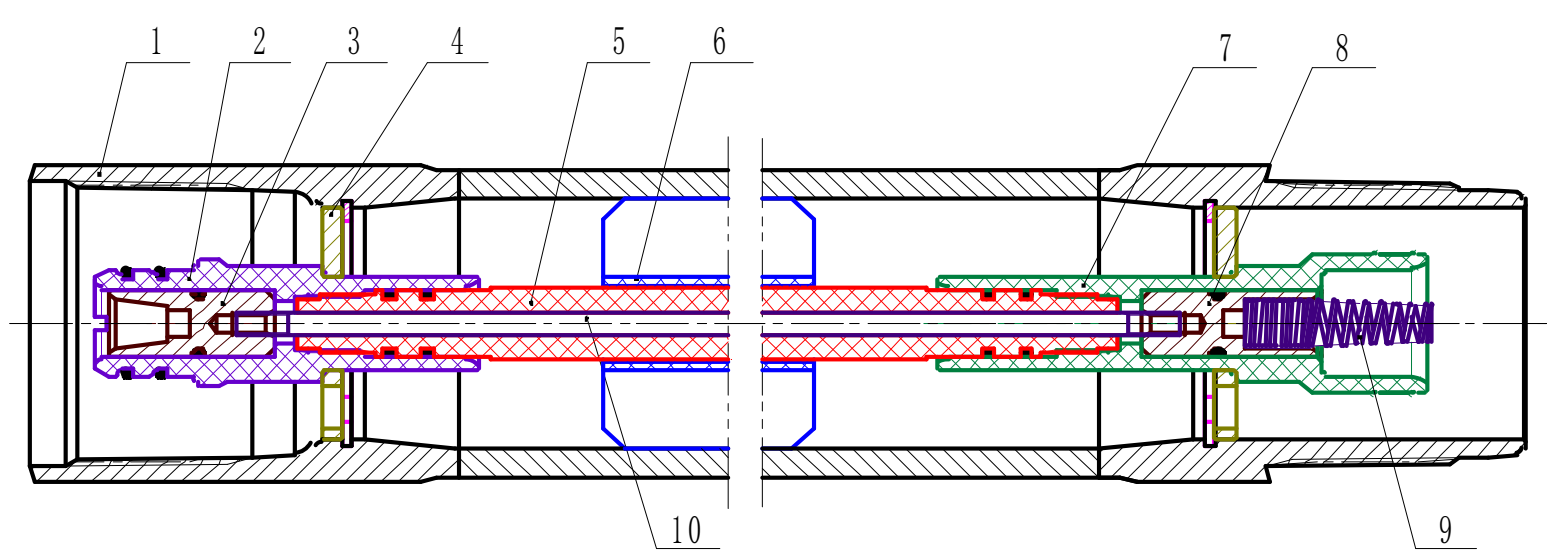

1. Outer pipe 2. Insulating pin joint 3. Conductor pin joint 4. Locating retainer ring 5. Conduit 6. Stabilizer 7. Insulating box joint 8. Conductor box joint 9. Variable-diameter spring 10. Copper conductor

Fig. 3 Structure Diagram of Center Cable Drill Rod

\section{Study on Critical Factors Influencing Signal Transmission}

Signal transmission device of measurement-while-drilling tool transmits signals from downhole inclinometer to display device at hole top, which is indispensable to continuous monitoring and timely rectification of borehole track. Therefore, it is of great importance to make sure the reliability of signal transmission device of measurement-while-drilling tool. During directional drilling, downhole motor is a downhole dynamic drilling tool adopting high-pressure flushing fluid to transfer power. To ensure enough input pressure of downhole motor, pressure loss during transmission of high-pressure fluid in drill rod shall be minimized. In order to minimize downhole signal attenuation, resistance of center cable device shall be minimized, and the signal transmission device shall be free of high-pressure aqueous medium during signal transmission to avoid serious signal attenuation which will interrupt signal transmission. Therefore, in directional drilling system, there are two critical factors influencing signal transmission: leakproofness and electroconductivity.

\section{Leakproofness of the system}

If high-pressure fluid flows into the signal transmission device, signal transmission will be interrupted. Therefore, it is important to make sure that the signal transmission device is well sealed. As shown in Fig. 3, in this system, there are two parts which shall be sealed: joint between conduit and plastic connector, where an O-shape sealing ring is adopted; joint between drill rods. On account of the high pressure of fluid around the equipment which may reach $8 \mathrm{MP}$, two sealing rings are necessary for each joint in practice. However, due to the bended borehole track, drill rods in the hole are also bended and so are the conduits in the drill rods, and hence the sealing between conduit and plastic connector is not reliable. To solve that problem, sealant is applied on the screw thread of conduit to strengthen the sealing effect.

Sealing of joints between drill rods is the most critical step for well functioning of the signal transmission device. Fig. 3 displays the joint with only two sealing rings applied, which always fails in application. The reason lies in the material and machining error of the plastic connector. Nylon was once adopted to seal plastic connector for its good toughness and stretching resistance. However, nylon is characterized by its water swelling and high hydroscopicity. The plastic 
connector was processed and assembled as per drawing. During drilling, nylon-applied joint immersed in high-pressure fluid in the inner bore of drill rod swells. Some box and pin joints with nylon applied on is hard to disassemble, and is easily broken once a drilling rig is used for disassembling. In addition, it is impracticable to reuse the box and pin joints with nylon applied on for another hole drilling because the joint covered with swelled nylon is too oversized to be plugged into the hole, and is easily deformed once a drilling rig is used for plug-in. The above phenomena occur from time to time. As a coping approach, the nylon-applied joint has been downsized and immersed in water for above 24 hours till the nylon stops swelling, before being assembled. The above approach, though qualified for sealing effect, is cumbersome and inefficient and adds a lot of check work to identify dimensions of the nylon-applied joints which are always in great difference.

To solve the above problem, glass fiber reinforced nylon (GFPA), a polymer of nylon and glass fiber, with good affinity with nylon, was adopted to reduce the hydroscopicity of nylon. However, cracking and fracture occurred on GFPA-applied drilling tools operating in the range of $1000 \mathrm{~m}-2000 \mathrm{~m}$, due to its low joint toughness and notch impact strength.

Later, based on research and material analysis, high impact-resistant polypropylene was selected, which features low density, favorable heat resistance and mechanical properties, stable chemical properties and good processing moldability. Plastic connector was well sealed by this material without deformation or fracture during field use, indicating that plastic connector sealed by polypropylene meets the communication needs of horizontal directional cable drill rod.

\section{Electroconductivity of the system}

In signal transmission device, there are many factors affecting electroconductivity of the system which are mainly stainless steel joint and center conductor. Stainless steel joint connects drill rods for signal transmission. Resistance of stainless steel joint of measurement-while-drilling system needs to be minimized and shall be corrosion and rust resistant. $2 \mathrm{Cr} 13$ was once adopted as material of stainless steel joint. However, drill rod is used in underground coal mine where acidic substances exist in the air. Therefore, stainless steel joint is required to be of extremely high acid resistance. Moreover, components and parts of drill rod in measurement-while-drilling system shall be non-magnetic and magnetically non-conductive as far as possible. To improve the performance of stainless steel joint, $1 \mathrm{Cr} 18 \mathrm{Ni}$ Ti was adopted as material of stainless steel joint, which turned out to have better corrosion resistance with signal transmission smoothened. As the longest communication component of cable device of drill rod, center conductor is used for signal connection, of which the resistance shall be minimized. Therefore, copper conductor was considered. At first brass H59 was chosen, which, however, was prone to breakage due to poor toughness and would interrupt signal transmission, and therefore was replaced by brass H62. But Brass H62 was found prone to screw thread wear due to poor strength and was finally, through concrete analysis and study, replaced by lead brass HPB59-3 featuring extremely high machinability, self-lubricating property, electroconductibility and strength. Lead brass HPB59-3 was applied well without breakage or wear of thread in field use, meeting the requirements for signal transmission.

The critical technology of center cable drill rod is signal transmission, and the effect of signal 
transmission always depends on sealing effect. That is to say, as long as all drill rods are completely sealed to ensure that signal transmission device is prevented from high-pressure medium, signal transmission will be smoothened and directional drilling process will be easy to perform. However, in production process, unsatisfactory signal transmission always occurs due to improper selection of materials. In addition, machining error is another cause of mismatching between tolerance clearances of plastic connectors, resulting in seal failure and transmission failure. Therefore, all plastic connectors, especially those required to be sealed, shall be inspected and qualified before use, since signal transmission will be affected even when a single seal failure on a drill rod occurs. From the above, it is clear that joint sealing is important for signal transmission, to which more attention shall be paid.

\section{Conclusions}

The paper expounds the design, thermal treatment and pilot production of center cable drill rod for coal mine as well as the importance of leakproofness and electroconductivity of the rod for its performance, based on researches in inner structure and outer pipe design of the rod and study on critical factors influencing signal transmission along the rod. The paper proposes solutions to problems encountered in design and processing of center cable drill rod, and helps provide technical equipment for directional drilling of long borehole in underground coal mines and ensure safe and highly efficient mining.

\section{References}

[1] Jingwei Zeng. Analysis on securities investment in coal industry [J]. Sichuan Cement, 2015, 01: 68.

[2] Jian Chen, Hongjun Zhang, Wei Fan. Application of technology and equipment in gas drainage of large diameter and long borehole [J] . Safety in Coal Mines, 2004, 35(4) :12-15.

[3] Ningping Yao, Jie Zhang, Qiaoqiao Li. Research and Application of Horizontal Directional Drilling Technology in Underground Mine [J] . Coal Science and Technology, 2011, 39(10):53 — 57.

[4] Zhijun Shi, Ningping Yao, Genfei Ye. Construction Technology and Equipment for Gas Drainage Borehole Drilling in Underground Coal Mine [J] . Coal Science and Technology, 2009, $37(7): 1-4$. 